\title{
The Problems and Challenges in Biomedical Sciences
}

\author{
Bruce Alberts
}

Chancellor's Leadership Chair in Science and Education, University of California, San Francisco.

Distressed by the perverse incentives that have generated the current hyper-competitive biomedical research environment in the United States, four of us published an open-access article in April 2014 entitled: Rescuing US biomedical research from its systemic flaws (Alberts, B., Kirschner, Marc W., Tilghman, Shirley, and Varmus, H.; Proc. Natl. Acad. Sci. USA 111, 57735777 (2014)). As announced in our follow-up piece, Opinion: Addressing systemic problems in the biomedical research enterprise (Proc. Natl. Acad. Sci. USA 112, 1912-1913 (2015)), we have formed a 16-member steering committee to oversee a new website that is designed to collect suggestions for actions that can ameliorate the identified problems, as well as to highlight promising changes that are either underway or proposed (see http://rescuingbiomedicalresearch.org).

Despite widespread agreement concerning the problems, any substantial change in the system is bound to be controversial. Experiments are therefore needed. In my talk, I will outline some possible ideas for overcoming the inertia that prevents moving forward.

We are encouraging both national and international contributions to this effort, since the problems that we have described are by no means unique to the United States. . 